\title{
Alterações pigmentares pós-tratamentos estéticos em pessoas de pele negra
}

\author{
Aesthetical post-treatment pigmentary changes in black skin people
}

Recebido em: 01/08/2018

Aceito em: 14/01/2019

Catiane Perereira RABELLO; Juçara FRANCISCO; Karina Elisa MACHADO

Universidade do Vale do Itajaí - UNIVALI. Rodovia SC-401, 5025, Saco Grande, CEP 88032-005, Florianópolis, SC, Brasil.E-mail: karymachado@hotmail.com

\section{ABSTRACT}

The demand for black skin facial aesthetic treatments (Phototypes IV, V, and VI on the Fitzpatrick Scale) has grown considerably in recent years. In counterpoint to this growth, the cosmetics and cosmetic procedures available in the market, in part, do not meet the specific needs of this type of skin. The black skin presents anatomical, physiological, and pathological characteristics, different from the white skin, which allow cosmetic treatments and cosmetic products to trigger pigmentary disorders. This way, the objective of this work was to present the main pigmentary alterations after facial aesthetic treatments in people with black skin. The methodology used in this research was a descriptive bibliographical review with a qualitative approach. In this context, information was sought on black skin, pigment disorders, aesthetic facial procedures, and cosmetic products. The results showed that dermal dyschromia's origin in black skin, as in other skin types, are most often the consequence of an inflammatory response, but the higher phototypes are more predisposed to pigment alterations. Among these dyschromias, one may highlight melasma, hyperpigmentation, and post-inflammatory hypopigmentation. It was also observed that the treatments mentioned in this article (depigmenting agents, chemical peelings, microdermabrasion, and microagglutment) present the risk of causing pigmentary alterations in high phototypes, especially if this skin already presents some previous lesion. It is also worth noting that even with the development of new products and new technologies, the Aesthetics and Cosmetics market still needs to invest more in research and development of products aimed at this public, considering the particularities of this type of skin.

Keywords: black skin; pigmentary alterations; aesthetic treatments; cosmetic products.

\section{RESUMO}

A procura por tratamentos estéticos faciais destinados à pele negra (Fototipos IV, V e VI, na escala de Fitzpatrick) tem crescido vertiginosamente nos últimos anos. Em contraponto a esse crescimento, os procedimentos estéticos e cosméticos disponíveis no mercado, em parte, não atendem às necessidades específicas deste tipo de pele. A pele negra apresenta características anatômicas, fisiológicas e patológicas diferentes da pele branca, que possibilitam aos tratamentos estéticos e produtos cosméticos desencadearem desordens pigmentares. Neste contexto, o objetivo deste trabalho foi apresentar as principais desordens pigmentares, pós-tratamentos estéticos faciais, em pessoas de pele negra. O presente estudo caracterizou-se com uma revisão bibliográfica exploratória-descritiva com abordagem qualitative. Nesse context, foram buscadas informações a respeito da pele negra, das desordens pigmentares, dos procedimentos estéticos faciais e dos produtos cosméticos. Os resultados mostraram que a discromia dérmica originada na pele negra, assim como em outros tipos de pele é, na maioria das vezes, consequência de uma resposta inflamatória, porém os 
fototipos elevados são mais predispostos às alterações pigmentares. Entre discromias podem ser destacados o melasma, a hiperpigmentação e a hipopigmentação pós-inflamatória. Os tratamentos citados no presente artigo (agentes despigmentantes, peelings químicos, microdermoabrasão e microagulhamento) oferecem risco de ocasionar alterações pigmentares em fototipos elevados, principalmente se a pele apresentar algum tipo de lesão prévia. Assim, mesmo com o desenvolvimento de novos produtos e novas tecnologias, o mercado da Estética e Cosmética ainda precisa investir mais em pesquisa e desenvolvimento de produtos destinados a esse público, considerando as particularidades deste tipo pele.

Palavras-chave: pele negra; discromias; tratamentos estéticos; produtos cosméticos

\section{INTRODUÇÃO}

Nos últimos anos no Brasil, a procura por cuidados estéticos destinados especificamente para a pele negra tem crescido vertiginosamente, considerando tanto a busca por cosméticos, quanto por procedimentos estéticos. Nesse contexto, destaca-se que a grande parte da população brasileira se considera de pele parda ou negra, e que, segundo dados do Instituto Brasileiro de Geografia e Estatística (IBGE), a população negra representa a maior parte da população brasileira (1).

Os tratamentos estéticos/cosméticos destinados à pele negra necessitam ser diferenciados e específicos, pois esse tipo de pele apresenta características anatômicas, fisiológicas e patológicas singulares. Outrossim, a definição de pele negra também possui variações; e na tentativa de uma uniformização do que é considerado, dermatologicamente, como pele negra, é utilizado o sistema de classificação chamado de Escala Fitzpatrick. Essa escala foi criada em 1976 pelo médico norte-americano Thomas B. Fitzpatrick e classifica a pele em fototipos de I a VI, a partir da capacidade de cada indivíduo apresenta em se bronzear, assim como, sensibilidade e vermelhidão da pele, quando essa é exposta ao sol $(2,3)$.

De acordo com a Escala de Fitzpatrick, a pele negra foi inicialmente definida como fototipo IV; entretanto, devido às variações de tonalidade, esta foi subdivida nos fototipos IV, V e VI, que raramente ou nunca queimam, quando expostos ao sol, e que bronzeiam com facilidade (3). Essa singularidade da pele negra é devida às suas características anatômicas, fisiológicas e patológicas. De acordo com Ifould e cols (2015), indivíduos com pele negra possuem maior produção sebácea e sudorífera, e os sinais de envelhecimento não aparecem até os quarenta anos (4).

Franquilino (2015), por sua vez, relatou que a pele negra apresenta uma maior produção de melanina e predisposição à formação de discromias (5). Nesta perspectiva, o trabalho de Alchorne e Abreu (2008) corroborou esta afirmação, pois destacou que grande parte das disfunções estéticas que acometem a pele negra são de origem pigmentar e de difícil de tratamento (6). Dentre as disfunções estéticas mais comuns na pele negra, os autores evidenciaram o melasma, a hiperpigmentação e a hipopigmentação pós-inflamatória (6).

O melasma caracteriza-se pelo surgimento de manchas escuras ou acastanhadas, geralmente, nas regiões malar, centro facial e mandibular, ocorrendo mais frequentemente nas mulheres, devido à ação hormonal (7). O melasma pode afetar qualquer raça ou fototipo, porém esta desordem acomete com mais frequência os asiáticos, hispânicos e os fototipos IV, V e VI (3).

A hipopigmentação e a hiperpigmentação pós-inflamatória, são consequência de uma resposta fisiopatológica a um trauma, podendo atingir ambos os sexos e todos os fototipos; porém os fototipos IV, V e VI podem ser os mais acometidos, apresentando pigmentação mais escura ou mais clara na pele, sendo estas de difícil tratamento (7).

Ainda, equipamentos e tratamentos estéticos também podem apresentar desordens pigmentares, como efeito colateral, principalmente quando aplicados por um profissional não habilitado que não conheça adequadamente os fenômenos inflamatórios da pele negra. Tanto os tratamentos estéticos como os cosméticos, se não utilizados corretamente, podem agravar as disfunções estéticas da pele negra, deixando ci- 
catrizes inestéticas e discromias, além de causar dano psicológico (8).

Nesta perspectiva, o objetivo deste trabalho consistiu em apresentar as principais alterações pigmentares pós-tratamentos estéticos faciais em pessoas de pele negra.

\section{MÉTODO}

Trata-se de uma revisão bibliográfica exploratória-descritiva com abordagem qualitativa. Para atender ao objetivo do estudo, as produções científicas referentes ao tema foram pesquisadas em livros e nos bancos de dados das bibliotecas eletrônicas Bireme, LILACS, SciELO, Pubmed e Periódicos CAPES, no período, preferencialmente, de 2008 a 2018. Os descritores utilizados para a seleção foram: procedimentos estéticos, pele negra, alterações pigmentares, melasma, hiperpigmentação e hipopigmentação pós-inflamatória. As estratégias utilizadas para inclusão dos artigos neste estudo foram artigos de pesquisas com estudos in vivo e in vitro, de revisão e artigos publicados nas versões inglês, espanhol e português disponíveis por completo nas bases eletrônicas. Os critérios de exclusão utilizados foram artigos repetidos, artigos incompletos e artigos e que não representavam a temática.

\section{RESULTADOS E DISCUSSÃO}

Pele negra. Segundo a Escala de Fitzpatrick, o tom da pele pode ser dividido em seis fototipos diferentes, de acordo com a reação cutânea, quando a pele está exposta a radiação solar, sendo o fototipo IV, inicialmente considerado a pele negra, que nunca queima e sempre bronzeia (3). Entretanto, devido ao fato de a pele negra abranger diferentes graduações de tonalidades, a escala foi subdividida nos fototipos IV, V e VI (Tabela 1), incluindo aqueles tipos que que raramente ou nunca queimam quando expostos ao sol, e que bronzeiam com facilidade (6).

Tabela 1. Fototipos Cutâneos de acordo com a Escala de Fitzpatrick (7)

\begin{tabular}{|l|l}
\hline Tipo de Pele & Descrição \\
\hline Tipo I & Pele muito clara, sempre queima, nunca bronzeia. \\
\hline Tipo II & Pele clara, sempre queima e algumas vezes bronzeia. \\
\hline Tipo III & Pele menos clara, algumas vezes queima e sempre bronzeia. \\
\hline Tipo IV & Pele morena clara, raramente queima e sempre bronzeia. \\
\hline Tipo V & Pele morena escura, nunca queima e sempre bronzeia. \\
\hline Tipo VI & Pele negra, nunca queima e sempre bronzeia. \\
\hline
\end{tabular}

A pele negra possui algumas particularidades em sua estrutura anatômica, fisiológica e patológica, que a diferencia das outras tonalidades de pele (9). Uma das características mais perceptíveis, neste tipo de pele, é a maior produção de melanina, responsável pela coloração do tecido cutâneo (5).

Estruturalmente o tegumento cutâneo dos indivíduos negros, apresenta algumas diferenças das outras raças, caracterizado pela maior presença de glândulas sudoríparas (apócrinas e écrinas) e maior número de vasos sanguíneos, desencadeando uma maior predisposição a hiperpigmentações. Porém ainda existem poucos estudos relacionados ao tema $(6,10)$.
Em seu trabalho, Franquilino (2015) relatou que a pele negra é mais oleosa na zona $\mathrm{T}$, apresentando ressecamento na área dos olhos, sendo considerada, de maneira geral, como uma pele mista (5). Batistela (2007) destacou que quando comparada com outras raças, a pele negra apresenta maior perda de água, o que a torna uma pele mais ressecada (10).

Não existe diferença na espessura da derme entre negros e brancos, porém existem algumas particularidades nos aspectos celulares (11). Os fibroblastos na pele negra são maiores, bi ou multinucleados, em maior número e hiperativos; as fibras colágenas são menores e dispostas mais para- 
lelamente à epiderme e os macrófagos são maiores e mais numerosos (5).

Associado a isso, na pele negra as camadas do tecido cutâneo encontram-se fortemente unidas em uma estrutura rígida, fazendo com que se torne mais firme e resistente ao surgimento de rugas, flacidez e hidrolipodistrofia ginóide (5).

Pode ser ainda salientado que a pele negra apresenta maior susceptibilidade ao surgimento de cicatrizes do tipo queloide quando lesionadas (4). Além disto, outras alterações aparecem com maior frequência neste grupo étnico, como o melasma, a hiperpigmentação pós-inflamatória, a ptiríase alba, a hipomelanose gutata idiopática, o vitiligo e as acromias secundárias, que podem ser recorrentes de doenças ou outro tipo de procedimento, como esfoliações químicas, crioterapia ou corticoterapia intralesional (6). Dentre as alterações pigmentares mais comuns na pele negra, podem ser destacados o melasma, a hiperpigmentação e a hipopigmentação pós-inflamatória (12).

\section{Tratamentos Estéticos Faciais em Pele} Negra. Tratar a pele faz parte de uma vida saudável de todos os indivíduos. Nesse cenário, a indústria cosmética vem se preocupando em oferecer procedimentos estéticos e cosméticos, com resultados satisfatórios e relativamente imediatos, para o tratamento de diversas disfunções estéticas, como: gordura localizada, flacidez, hidrolipodistrofia ginoide, estrias ou alterações pigmentares (13).

Para o sucesso de qualquer tratamento, o conhecimento que o profissional tem sobre anatomia, fisiologia e patologia, bem como sua qualificação e atualização é imprescindível para a seleção do tratamento mais adequado (14). Neste contexto, os tratamentos estéticos e cosméticos consistem basicamente em manobras manuais, uso de equipamentos eletroterápicos e uso de cosméticos.

Diversos tratamentos estéticos realizados em fototipos elevados com a finalidade de melhorar a aparência, tratar a pele ou rejuvenescer, acabam gerando uma agressão nas primeiras camadas da epiderme, deixando o tecido sensibilizado e, consequentemente, gerando uma resposta inflamatória local, que pode provocar alteração na atividade dos melanócitos, aumentando desta forma, a quantida- de de melanina produzida, gerando assim alterações pigmentares (2).

Assim, dentre os diversos tratamentos estéticos e cosméticos faciais, foram selecionados os seguintes: agentes despigmentantes, peelings químicos, microdermoabrasão e microagulhamento, devido à importância que os mesmos ocupam no mercado da Estética e Cosmética.

Despigmentantes. São substâncias capazes de mudar a pigmentação da pele. Geralmente agem sobre os melanócitos, nas diferentes fases da melanogênese ou na transferência da melanina para o queratinócitos, sendo eficientes em diversos distúrbios funcionais dos melanócitos, entre eles, o melasma (15).

Conceitualmente, são produtos que auxiliam na atenuação de algumas hiperpigmentações, podendo ser encontrados em diversas formas de apresentação como pomadas, cremes, loções, entre outras. O tratamento para hipercromias consiste no uso dessas substâncias que deverão agir no local específico do corpo, por meio de diferentes mecanismos de ação (16).

$\mathrm{O}$ agente despigmentante ideal deve apresentar várias propriedades, que incluem uma ação eficaz com pouco ou nenhum efeito colateral, agindo em uma ou mais etapas do processo melanogênico (12).

De acordo com Rodrigues (2016), no tratamento das discromias podem ser utilizados diferentes tipos de despigmentantes, considerando que sua ação possa ocorrer através de diferentes mecanismos (17). Dentre os despigmentantes mais utilizados no tratamento das alterações pigmentares cutâneas, pode ser destacada a hidroquinona, um agente inibidor da tirosinase, enzima chave no processo na melanogênese. A hidroquinona ainda pode ser associada com alguns antioxidantes, para melhorar a eficácia do tratamento. Entretanto seu uso pode originar reações adversas como dermatite de contato e hipopigmentação irreversível.

Peeling químico. Também conhecido como quimioesfoliação, quimiocirurgia ou dermopeeling. Consiste em um tratamento que utiliza agentes esfoliantes sobre a pele, podendo estes serem utilizados isoladamente ou em associação, como objetivo de ocasionarem a destruição parcial da epiderme ou derme, estimulando a regeneração tecidual (18). 
O peeling químico é a ação de substâncias químicas sintéticas ou vegetais sobre a pele, proporcionando intensa renovação cellular, Geralmente as substâncias utilizadas são ácidos orgânicos em diferentes concentrações (19). Contudo, a utilização de uma concentração elevada de ácidos, como glicólico e o retinoico, deve ser evitada em pele negra, pois estes ácidos podem provocar discromias, como manchas esbranquiçadas nas regiões onde a pele é mais fina.

Os peelings, tanto superficiais quanto médios, também podem ocasionar hiperpigmentações em indivíduos dos fototipos IV, V e VI, independente do agente esfoliante utilizado, podendo estas ser desencadeadas espontaneamente ou por exposição solar mínima durante a fase eritematosa (18).

Microdermoabrasão. É caracterizada como uma técnica de esfoliação (peeling mecânico), considerada indolor, não invasiva e passível de controle. Esta técnica pode ser utilizada nos tratamentos de clareamento epitelial, devido sua ação esfoliativa sobre a camada córnea (2). Contudo, os fototipos IV, V e VI podem sofrer hiperpigmentação, caso a técnica não seja aplicada corretamente.

Microagulhamento. É uma técnica na qual são realizadas microperfurações na pele, com centenas de agulhas de aço inoxidável, alinhadas e estéreis, presas a um rolo de polietileno. A técnica compreende a realização de movimentos de ida e volta, com objetivo de obter pequenas perfurações na pele. Outra função do microagulhamento é promover a permeação dos ativos na pele, também conhecido como drug delivery, podendo associar, à técnica, cosméticos tópicos clareadores (20).

Quando a pele negra é submetida a traumatismo, o risco de gerar hipercromia é maior, portanto deve haver um cuidado maior na realização deste procedimento em indivíduos negros (21).

Desordens pigmentares recorrentes de tratamentos estéticos na pele negra. Grande parte das alterações percebidas em pele negra estão relacionadas às alterações pigmentares (6). Esses distúrbios geralmente são difíceis de serem tratados e, entre estes, destacam-se: o melasma, a hiperpigmentação e hipopigmentação pós-inflamatória.
Melasma. Também conhecido como cloasma, é uma disfunção estética ou pigmentária cutânea "possuindo características simétricas, com bordas irregulares, mas com limites nítidos e máculas acastanhadas e de intensidades diferentes" (22). Pode atingir tanto homens como mulheres, sendo diagnosticada principalmente em mulheres, devido à ação hormonal, acometendo diversas partes do corpo, entre elas a face, o pescoço, o colo e o antebraço.

A palavra melasma deriva do grego "melas", que significa "negro" (23). Melasma é uma hipermelanose cutânea simétrica, adquirida, de ocorrência comum, resultante de uma desregulação do sistema pigmentar da pele e que ocorre predominantemente na face, sendo intensificada pela radiação solar $(24,25)$. O melasma é considerado uma dermatose que causa aumento de pigmentação da pele (26). Outro trabalho apresenta que o melasma caracteriza-se como uma mácula de coloração marrom, tendo em média de 1 a $2 \mathrm{~cm}$, podendo surgir nas faces, na região frontal e nas têmporas, sendo mais comum em mulheres, por conta do uso de anticoncepcionais e cosméticos fotossensibilizantes (19).

O melasma é uma patologia frequente e, embora possa acometer ambos os sexos e todas as raças, favorece fototipos intermediários, indivíduos de origem oriental ou hispânica. O distúrbio estético costuma incomodar muito quem o tem, trazendo transtornos psíquicos e emocionais. Para tratamento são utilizados peelings, ácidos e cosméticos despigmentantes; em indivíduos de pele negra, esses tratamentos também podem estimular o aparecimento de distúrbios pigmentares (27).

Hiperpigmentação e hipopigmentação pós-inflamatória. A hiperpigmentação pós-inflamatória manifesta-se como áreas irregulares com pigmentação escura, originadas em regiões com inflamação prévia. Atinge principalmente a face, gerando angústia nos indivíduos acometidos; entretanto, esta hipercromia pode afetar qualquer região do corpo (2).

A hiperpigmentação pós-inflamatória caracteriza-se como um estágio secundário, podendo ocorrer em quase todo processo inflamatório (28). Sua distribuição acontece de forma variada, pois depende do processo inflamatório inicial. A origem desta 
desordem pigmentar secundária, está relacionada à estimulação dos melanócitos pelos mediadores inflamatórios do processo primário.

A hiperpigmentação pós-inflamatória acontece devido ao aumento na produção da melanina e quando atinge a epiderme, apresenta uma coloração que varia entre o marrom claro e o escuro, dependendo da quantidade de pigmento produzida na pele, quando em nível dérmico afeta os melanófagos, conferindo uma cor cinza azulada à pele $(18,29)$. Essas manchas podem surgir devido a fatores como envelhecimento, alterações hormonais, inflamações, alergias e exposição solar, dentre outros (29).

As hipocromias são caracterizadas como manchas com um tom mais claro que a pele, sendo causadas pela diminuição da melanina epidérmica. Esta disfunção também pode ser denominada como hipopigmentação, hipomelanose ou leucodermias (7).

As lesões hipocrômicas, se manifestam por meio de uma concentração diminuída de melanina, consequência da ausência dos melanócitos e problemas na formação dos melanossomas, originando manchas mais claras, como a ptiríase versicolor, podendo ser localizadas ou generalizadas. Ocorrem por hereditariedade, congenitamente, ou podem ser adquiridas, devido a processos inflamatórios (29).

O tratamento dessas desordens estéticas é difícil e lento, sendo fundamental tratar a doença inflamatória que originou o distúrbio pigmentar. Para a hiperpigmentação são utilizados agentes despigmentantes e esfoliações químicas superficiais, que devem ser utilizados com cautela (6). As opções para a hipopigmentação incluem os corticosteroides e os psoralenos associados à luz ultraviolet A. Contudo, em indivíduos de pele negra, esses tratamentos também podem estimular o aparecimento dos distúrbios estéticos.

Tratamentos estéticos faciais em pele negra $\mathbf{X}$ desordens pigmentares. As discromias dérmicas originadas na pele negra são, na maioria das vezes, consequência de uma resposta inflamatória, assim como em outros tipos de pele; porém, os fototipos altos (IV, V e VI) são mais predispostos às alterações pigmentares. $\mathrm{O}$ aumento na produção de melanina, em decorrência da estimulação direta ou indireta, é uma forma da pele se preservar, se protegendo contra as agressões solares ou inflamações, por exemplo (29). Desta forma, quando a pele é exposta à irradiação, os melanossomas se reagrupam em torno do núcleo, a fim de proteger o material genético da célula e, assim, promoverem o escurecimento da mesma. Além disto, os melanócitos também são células responsáveis pela promoção da coloração da pele, pelos e cabelos. Nesta perspectiva, a melanina é um pigmento que promove fotoproteção, agindo como um filtro solar natural, difratando ou refletindo a radiação solar.

Quando os melanócitos sofrem estimulação seja por fatores internos ou externos, ocorre uma produção excessiva de melanina epidérmica ou dérmica, sendo originadas, assim, as desordens hipercrômicas (29); e os indivíduos de pele negra apresentam naturalmente, uma maior atividade dos melanócitos (4-6).

Neste contexto destaca-se o mercado de Estética e Cosmética, e seus diversos tratamentos estéticos faciais. Os procedimentos abordados (agentes despigmentantes, peelings químicos, microdermoabrasão e microagulhamento), embora se tratem de procedimentos diferenciados, apresentam alguns pontos em comum, como o fato de serem muito utilizados em diferentes procedimentos faciais e, principalmente, a inexistência de literatura adequada e protocolos específicos para indivíduos de fototipos elevados (IV, V e VI). Na maioria das vezes a utilização para pele negra (fototipos IV, V e VI) é apresentada como restrição de uso e/ou com a observação de que deve ser utilizado com cautela $(2,12-21)$.

Assim, estes tratamentos quando aplicados em fototipos elevados (IV, V e VI) sem o devido conhecimento sobre a anatomia, fisiologia e patologia deste tipo de pele, podem provocar alterações na atividade dos melanócitos, aumentando desta forma a quantidade de melanina produzida, gerando assim o aparecimento de discromias, por esta razão a atenção deve ser redobrada na realização dos procedimentos em indivíduos negros (fototipos IV, $\mathrm{V}$ e VI) $(2,6,18-19,22,28,29)$. 
Tabela 2. Restrições de uso e/ou a observação de procedimentos Estéticos e Cosméticos em Indivíduos de pele negra (Fototipos IV a VI).

\begin{tabular}{|c|c|l|}
\hline Referência & Método & \multicolumn{1}{c|}{ Restrição e/ou Observação } \\
\hline$(17)$ & $\begin{array}{c}\text { Despigmentante } \\
\text { Hidroquinona }\end{array}$ & $\begin{array}{l}\text { Pode gerar hipopigmentação irreversivel em fototipos IV, V e VI. } \\
\text { Evitar o uso de hidroquinona isoladamente em fototipos IV, V e VI. }\end{array}$ \\
\hline$(21)$ & Microagulhamento & $\begin{array}{l}\text { Risco de gerar hipercromia em fototipos IV, V e VI. } \\
\text { Os fototipos IV, V e VI, podem sofrer hiperpigmentação, caso a técnica não } \\
\text { seja aplicada corretamente. }\end{array}$ \\
\hline \multirow{2}{*}{ (18) } & Microedermoabrasão & $\begin{array}{l}\text { Tanto os peelings superficiais quanto médios podem ocasionar hiperpigmen- } \\
\text { tações em indivíduos dos fototipos IV, V e VI; } \\
\text { Independente do agente esfoliante utilizado pode ser desencadeada dis- } \\
\text { cromias espontaneamente, em fototipos IV, V e VI, ou caso haja exposição } \\
\text { solar mínima durante a fase eritematosa; } \\
\text { Deve-se evitar o uso de hidroquinona isoladamente em fototipos IV, V e VI; }\end{array}$ \\
\hline
\end{tabular}

Os tratamentos estéticos faciais na pele negra (fototipos IV, V e VI) só devem ser realizados quando a mesma estiver devidamente hidratada, ou seja, sem apresentar um aspecto acinzentado ou escoriações, a fim de minimizar a possiblidade de reações inflamatórias (2,5-6,9,10-14). Também é importante que cada pele seja avaliada de forma única, para a escolha do tratamento adequado.

Ainda não existem. no Mercado Brasileiro, planos de tratamentos faciais específicos para pele negra (fototipos IV, V e VI) e cada profissional deve fazer as adaptações que julgar necessárias, tais como reduções nas concentrações de peelings químicos e despigmentantes, para evitar possíveis alterações pigmentares, bem como manusear corretamente os equipamentos eletroperáticos utilizados no tratamento.

Na Tabela 2 são apresentadas algumas restrições de uso e/ou as observações, abordadas.

\section{CONCLUSÃO}

Foi possível observar que as alterações pigmentares pós-tratamentos estéticos são, na sua maioria, de origem inflamatória, e têm como consequência, desordens pigmentares. Os tratamentos disponíveis (agentes despigmentantes, peeligns químicos, microdermoabrasão e microagulhamento) oferecem risco de ocasionar alterações pigmentares em fototipos elevados (IV, V e VI), principalmente se apele apresentar algum tipo de lesão prévia.

Outro ponto observado foi a carência de estudos relacionados ao tema abordado. Desta forma, há necessidade de mais pesquisas referentes às desordens pigmentares na pele negra, com o objetivo de elaborar diferentes formas de cuidados no planejamento para o atendimento de indivíduos negros (fototipos IV, V e VI), considerando as peculiaridades deste tipo de pele e a necessidade de cuidados específicos em um tratamento facial, Dessa forma, o professional poderá escolher ou realizar o tratamento adequado, visando minimizar o risco de efeitos colaterais. Por fim, destaca-se a necessidade de atualização e capacitação, por parte do profissional, para execução de procedimentos adequados nos atendimentos de indivíduos negros (fototipos IV, V e VI). 


\section{REFERÊNCIAS}

1. Saraiva A. População chega a 205,5 milhões, com menos brancos e mais pardos e pretos. Brasília: Estatística Sociais. 2017. Disponível em: https://nacaomestica.org/blog4/wp-content/uploads/2018/07/ IBGE-_-Ag\%C3\%AAncia-de-Not\%C3\%ADcias-_-Popula $\%$ C3\%A7\%C3\%A3o-chega-a-2055-milh\%C3\%B5es-com-menos-brancos-e-mais-pardos-e-pretos. pdf. Acessado em: 15 mar. 2018.

2. Hill P. Milady: Microdermoabrassão. 2a. ed. São Paulo: Cengage. 2016.

3. Lopes DS. A utilização do ácido tranexâmico no tratamento de melasma. Rev. Cien. FHO. 2017;5(1):37-43.

4. Ifould J, Forsythe-Conroy D, Whittaker M. Técnicas em estética, 3a. ed. Porto Alegre: Artmed. 2015.

5. Franquilino E. Pele negra: conheças as particularidades, necessidades nuances do fototipo que corresponde a mais da metade da população brasileira. Cosmet. Toiletries. 2015;27(18):18-21.

6. Alchorne MMA, Abreu MAMM. Dermatologia na pele negra. An. Bras. Dermatol. 2008;83(1):7-20.

7. Ribeiro D. Recursos técnicos em estética. São Paulo: Difusão. 2013.

8. Monteiro EO. Pele morena: laser e tecnologia correlata. RBM. 2012;4:18-23.

9. Frangie CM, Botero AR, Hennessey C, Lees DM, Sanford B, Shipman F, Wurdinger V, Higuchi CT. Milady Cosmetologia: Ciências gerais, da pele e das unhas. São Paulo: Cengage. 2016.

10. Batistela MA. Abordagens no estudo do envelhecimento cutâneo em diferentes etnias. RBFarma. 2007;88(2):59-62.

11. D’Elia MPB. Avaliação comparativa da ancestralidade em mulheres com melasma facial: um estudo transversal. [Dissertação]. Botucatu: Universidade Estadual Paulista. 2015.

12. Frazon MA. Efeito do extrato de cascas de Rauvolfia sellowii Müll Arg. e de Himatanthus lancifolius (Müll) Arg. e dos alcaloides uleína e ioimbina na melanogênese. [Dissertação]. Curitiba: Universidade Federal do Paraná. 2013.

13. Mateus A, Palermo E. Cosmiatria e Laser: prática no consultório médico. São Paulo: AC farmacêutica. 2015

14. Michalun MV, Dinardo JC. Ingredientes para cosmética e cuidado da pele. São Paulo: Cengage Learning. 2016.

15. Sponchiado G. Avaliação da eficácia e segurança da Malva sylvestris com potencial atividade para tratamento de disfunções da pele. [Tese]. Curitiba: Universidade Federal do Paraná. 2015.
16. Nicoletti MA. Hipercromias: aspectos gerais e uso de despigmentantes cutâneos. Cosmet. Toiletries. 2002;14:46-51.

17. Rodrigues B. Estudo comparativo do tratamento da hiperpigmentação axilar utilizando ativos cosméticos e eletroterapia. [Trabalho de Conclusão de Curso]. Rio Grande do Sul: Universidade de Santa Cruz do Sul, Santa Cruz do Sul. 2016.

18. Kede MPV, Sabatovich O. Dermatologia estética. São Paulo: Atheneu. 2004.

19. Gomes RK, Damazio MG. Cosmetologia: descomplicando os princípios ativos. 3a. ed. São Paulo: Livraria Médica Paulista. 2009.

20. Lemos ACC. Estudo histomorfométrico, ultraestrutural e da expressão de Wnt1, WIF-1 e ASIP na pele com melasma em comparação com a pele sã perilesional e retroauricular. [Dissertação]. Botucatu: Universidade Estadual Paulista. 2017.

21. Tiburtino KMS, Vidal GP. Ação do dermaroller nas hipercromias dérmicas: revisão de literatura. Temas Saud. 2017;17(2):149-158.

22. Marques SS. Tipos de melasma e seus tratamentos. Rev. UNIPLAC. 2018;6(1):24-30.

23. Guevara IL, Pandya AG. Safety and efficacy of $4 \%$ hydroquinone combined with $10 \%$ glycolic acid, antioxidants, and sunscreen in the treatment of melisma. Int J Dermatol. 2003;42(12):966-972. DOI: 10.1111/j. 1365-4632.2003.02017.x

24. Morais OO, Souza MCS, Costa IMC, EFL Lemos, CM Gosmes, CDR Paula. O uso de lasers ablativos no tratamento do melasma facial. An. Bras. Dermatol. 2013;88(2):240-245.

25. Arefiev KLB, Hantash BM. Advances in the treatment of melasma: a review of the recent literature. Dermatol Surg. 2012;38(7):971-984. DOI: 10.1111/j. 1524-4725.2012.02435.x

26. Mazon VFP. Utilização do laser no tratamento do melasma. Rev. Maieut. 2017;1(1):75-84.

27. Miot LDB. Estudo comparativo morfofuncional de melanócitos em lesões de melasma. An. Bras. Dermatol. 2007;82(6):529-534. DOI: 10.1590/S036505962007000600005

28. Pedrosa TN. Avaliação do potencial de derivados do ácido kójico sobre a melanogênese e o envelhecimento cutâneo. [Dissertação]. Manaus: Universidade Federal do Amazonas. 2013.

29. Gonchorosk DD, Côrrea GM. Tratamento de hipercromia pós-inflamatória com diferentes formulações clareadoras. Infarma. 2005;17(3/4):84-86. 